Pacific Journal of Mathematic 


\section{A MINIMAL BOUNDARY FOR FUNCTION ALGEBRAS}

\section{ERRETT BISHOP}

1. Introduction. An algebra $\mathfrak{A}$ of continuous functions on a compact Hausdorff space $C$ will be understood to be a set of complex-valued functions on $C$ which is closed under the operations of addition, multiplication, and multiplication by complex numbers. The algebra $\mathfrak{A}$ is called separating if to any two distinct points of $C$ there exists a function in $\mathfrak{A}$ which takes distinct values at the given points. The norm $\|f\|$ of a continuous function $f$ on a compact space is defined to be the maximum absolute value of the function. The algebra $\mathfrak{A}$ is thus a normed algebra. $\mathfrak{U}$ is called a Banach algebra if it is complete with respect to its norm, i.e., if the limit of every uniformly convergent sequence of elements of $\mathfrak{A}$ is in $\mathfrak{A}$.

An important theorem of Šilov (see [5], p. 80) asserts that if $\mathfrak{A}$ is a separating algebra of continuous functions on a compact Hausdorff space $C$ then there is a smallest closed subset $S$ of $C$ having the property that every function of $\mathfrak{A}$ attains its maximum absolute value at some point of $S$. This set is called the Šilov boundary of $\mathfrak{A}$. A simple example is obtained by taking $C$ to be a compact subset of the complex plane and $\mathfrak{A}$ to be the set of all continuous functions on $C$ which are analytic at interior points; in this case the Šilov boundary of $\mathfrak{A}$ coincides with the topological boundary of $C$.

Given a separating normed algebra $\mathfrak{A}$ of continuous functions on a compact space $C$, it seems natural to ask, in view of Šilov's theorem, whether there exists a smallest subset $M$ (not necessarily closed) of $C$ having the property that every function in $\mathfrak{A}$ attains its maximum absolute value at some point of $M$. The answer in general is no. However, it will be shown (Theorem 1 below) that such a set $M$, called the minimal boundary of $\mathfrak{A}$, always exists if in addition it is assumed that $\mathfrak{U}$ is a Banach algebra and that there is a countable basis for the open sets of $C$, i.e., that $C$ is metrizable. An example will be given to show that the metrizability of $C$ is necessary.

If the minimal boundary $M$ exists, it is clear that the closure of $M$ is the Šilov boundary. An example will be given to show that $M$ need not be closed, so that $M$ in general is smaller than the Śilov boundary. This raises the question of the topological structure of $M$, which is answered (Theorem 2 ) by showing that $M$ is a $G_{\delta}$, i.e., a countable intersection of open sets.

Received December 1, 1958, in revised form February 23, 1959. The author holds a Sloan Foundation Fellowship. 
The next portion of the paper concerns the representation of bounded linear functionals on $\mathfrak{A}$ by measures. It is an easy consequence of the classical Hahn-Banach theorem and the Riesz representation theorem that any bounded linear functional $\varphi$ on $\mathfrak{A}$ of norm 1 can be represented by a (complex-valued, Borel) measure $\mu$ of norm 1 on the Šilov boundary $S$ of $\mathfrak{A}$, in the sense that $\varphi(f)=\int_{S} f d \mu$ for all $f$ in $\mathfrak{A}$. It is natural to conjecture that $\mu$ can actually be taken to be a measure on the minimal boundary $M$ of $A$. The author will devote a subsequent paper to a proof of this result and a consideration of related questions. Karl de Leeuw also has a proof of this result, based on work of Choquet [3]. In the present paper we prove a special case, which is needed to prove the general result and which will be sufficient for the applications considered here. This special case, Theorem 3 below, states that for any point $x$ in $C-M$ there exists a non-negative valued measure $\mu$ of norm 1 on $C-\{x\}$ such that $f(x)=\int f d \mu$ for all $f$ in $\mathfrak{A}$.

The final section is concerned with problems of approximation in one complex variable. Necessary and sufficient conditions are obtained on a compact set $C$ without interior of the complex plane that every continuous function on $C$ be uniformly approximable by rational functions whose poles lie in the complement of $C$. Mergelyan [6] has obtained sufficient conditions, of a different type, that the approximation be possible.

A summary of the results of this paper was given in [1].

\section{The minimal boundary.}

DEFINITION 1. Let $f$ be a continuous function on a compact space $C$. Then $S(f)$, the maximal set of $f$, consists of all points $x$ in $C$ such that $|f(x)|=\|f\|$.

DeFinition 2. Let $\mathfrak{A}$ be a separating algebra of continuous function on a compact space $C$. A subset $N$ of $C$ is said to bound $\mathfrak{A}$ if $N \cap S(f)$ is non-void for all $f$ in $\mathfrak{H}$. If the class of subsets of $C$ which bound $\mathfrak{A}$ contains a smallest set $M$, the set $M$ will be called the minimal boundary of $\mathfrak{A}$.

THEOREM 1. Let $\mathfrak{U}$ be a separating Banach algebra of continuous functions on a compact metrizable Hausdorff space $C$. Then $\mathfrak{i}$ has a minimal boundary $M$ and $M$ equals the subset $M_{0}$ of $C$ consisting of all $x$ in $C$ such that there exists $f$ in $\mathfrak{A}$ with $S(f)=\{x\}$

Proof. Let $N$ be an arbitrary subset of $C$ which bounds 2 . For 
each $x$ in $M_{0}$, there exists $f$ in $\mathfrak{A}$ with $S(f)=\{x\}$. Thus $\{x\} \cap N=$ $S(f) \cap N$ is non-void. Hence $x \in N$. Therefore $M_{0} \subset N$.

To show that $M_{0}$ is indeed the minimal boundary of $\mathfrak{A}$, it remains to prove that $M_{0}$ bounds $\mathfrak{A}$. It must therefore be shown that $M_{0} \cap S(f)$ is non-void for each $f$ in $\mathfrak{A}$. Let $f$ be given. Let $I$ be the class of all subsets $\gamma$ of $C$ such that there exists $f_{\gamma}$ in $\mathfrak{N}$ with $S\left(f_{\gamma}\right)=\gamma$. By Zorn's lemma, there is a subclass $\Gamma_{0}$ of $\Gamma$ which contains $S(f)$, which has the finite intersection property, and which has the property that no larger subclass of $\Gamma$ has the finite intersection property. Since $C$ is compact and since each $\gamma$ in $\Gamma_{0}$ is closed, the set $D=\bigcap_{\Gamma_{0}} \gamma$ is non-void and closed. Since there is a countable basis for the open sets of $C$, and since the family $\left\{C-\gamma: \gamma \in \Gamma_{0}\right\}$ of open sets covers $C-D$, there exists a sequence $\left\{\gamma_{n}\right\}$ from $\Gamma_{0}$ such that $\left\{C-\gamma_{n}\right\}$ covers $C-D$, i.e., such that $D=\bigcap \gamma_{n}$. Fix a point $x_{0}$ of $D$. Define

$$
f_{n}=\left[f_{\gamma_{n}}\left(x_{0}\right)\right]^{-1} f_{\gamma_{n}} .
$$

Clearly $S\left(f_{n}\right)=\gamma_{n}$ and $\left\|f_{n}\right\|=f_{n}\left(x_{0}\right)=1$. Thus the series $\sum_{n=1}^{\infty} 2^{-n} f_{n}$ converges uniformly on $C$ to a function $g$ in $\mathfrak{R}$ with $\|g\|=g\left(x_{0}\right)=1$. If $x \in C-\gamma_{k}$, then $|g(x)| \leqq \sum 2^{-n}\left|f_{n}(x)\right|<1$ since $\left|f_{n}(x)\right| \leqq 1$ for all $n$ and $\left|f_{k}(x)\right|<1$. Therefore $S(g) \subset \gamma_{k}$. Thus $S(g) \subset \bigcap \gamma_{k}=D$. Assume that $S(g)$ contains more than one point. Since $\mathfrak{A}$ separates points, there exists $h_{0}$ in $\mathfrak{A}$ which is not constant on $S(g)$. We may assume that the maximum of $\left|h_{0}\right|$ on $S(g)$ is 1 and that $h_{0}$ takes the value 1 at some point of $S(g)$. If we set $h=h_{0}+h_{0}^{2}$, it follows that the maximum of $|h|$ on $S(g)$ is 2 and that this maximum is attained only where $h_{0}$ takes the value 1 . Thus $|h|$ is not constant on $S(g)$. Therefore the set

$$
E=\{x: x \in S(g) \text { and }|h(x)| \geqq|h(y)| \text { for all } y \text { in } S(g)\}
$$

is a proper closed subset of $S(g)$.

Let $x_{1}$ be any point in $E$. Define the functions

$$
g_{0}=\left[g\left(x_{1}\right)\right]^{-1} g
$$

and

$$
h_{0}=\left[h\left(x_{1}\right)\right]^{-1} h \text {. }
$$

Thus $\left\|g_{0}\right\|=g_{0}\left(x_{1}\right)=1$ and $S\left(g_{0}\right)=S(g)$. Also $h_{0}\left(x_{1}\right)=1,\left|h_{0}(x)\right| \leqq 1$ if $x \in S(g)$, and $\left|h_{0}(x)\right|<1$ if $x \in S(g)-E$. Let $K=\left\|h_{0}\right\|$. For each positive integer $n$, let

$$
V_{n}=\left\{x: 1+2^{-n}(K-1) \leqq\left|h_{0}(x)\right| \leqq 1+2^{-n+1}(K-1)\right\} .
$$

Clearly $\bigcup V_{n}=\left\{x:\left|h_{0}(x)\right|>1\right\}$. Thus $V_{n} \cap S(g)=V_{n} \cap S\left(g_{0}\right)$ is void for each $n$. Therefore $\left|g_{0}(x)\right|<1$ for each $x$ in $V_{n}$. Since $V_{n}$ is compact, it follows that there exists a positive integer $p_{n}$ such that $\left|g_{0}(x)\right|^{p} \leqq 1 / 2$ 
for all $x$ in $V_{n}$. Since $\left\|g_{0}\right\| \leqq 1$, the series

$$
h_{0}+4(K-1) \sum_{n=1}^{\infty} 2^{-n} g_{0}^{n} n
$$

converges uniformly on $C$ to a function $k$ in $\mathfrak{A}$. We have

$$
k\left(x_{1}\right)=1+4(K-1) \sum_{n=1}^{\infty} 2^{-n}=1+4(K-1) .
$$

If $x \in S(g)-E$, then $\left|h_{0}(x)\right|<1$ and $\left|g_{0}(x)\right|=1$, so that $|k(x)|<1+4(K-1)$. If $x \in C-\bigcup V_{n}$, then $\left|h_{0}(x)\right| \leqq 1$ and $\left|g_{0}(x)\right| \leqq 1$, so $|k(x)| \leqq 1+4(K-1)$. If $x \in V_{j}$, then $\left|h_{0}(x)\right| \leqq 1+2^{-j+1}(K-1),\left|g_{0}(x)\right|^{p} n \leqq 1$ for all $n$, and $\left|g_{0}(x)\right|^{p_{j}} \leqq 1 / 2$, so that $|k(x)| \leqq 1+4(K-1)$. Therefore $k\left(x_{1}\right)=1+4(K-1)=$ $\|k\|$. Thus $x_{1} \in S(k)$ and $S(k)$ is disjoint from $S(g)-E$. Since $x_{1} \in S(g) \subset D=$ $\bigcap_{F_{0}} \gamma$, and since $S(k) \in \Gamma$, it follows from the maximality of $\Gamma_{0}$ with respect to the finite intersection property that $S(k) \in \Gamma_{0}$. Therefore $S(g) \subset \bigcap_{r_{0}} \gamma \subset S(k)$. Since $S(g)-E$ is non-void, this contradicts the fact that $S(g)-E$ is disjoint from $S(k)$. Therefore the assumption that $S(g)$ contains more than one point is false. Thus $S(g)$ consists of a single point $x_{0}$. It follows that $x_{0} \in M_{0}$. Since $S(g) \subset D=\bigcap_{\Gamma_{0}} \gamma \subset S(f)$, it follows that $x_{0} \in S(f) \cap M_{0}$. Thus $S(f) \cap M_{0}$ is non-void, as was to be proved.

We now give an example to show that Theorem 1 fails if $C$ is not metrizable. Let $I$ denote the unit interval $[0,1]$ with the usual topology. Let $\Gamma$ be an uncountable set. Let $C$ consist of all families $x=\left\{x_{\alpha}\right\}_{a \in z}$ with $x_{\alpha} \in I$ for each $\alpha$. Thus $C$ is the Cartesian product of an uncountable number of intervals, and is therefore compact. Let $\mathfrak{A}$ consist of all continuous functions $f$ on $C$ which have the property that there exists a countable subset $\Delta$ of $\Gamma$ such that $f(x)=f(y)$ whenever $x$ and $y$ are points in $C$ such that $x_{\alpha}=y_{\alpha}$ for all $\alpha$ in $\Delta$. It is easy to see that $\mathfrak{A}$ is a separating Banach algebra of continuous functions on $C$. By the Stone-Weierstrass theorem it follows that $\mathfrak{A}$ consists of all continuous functions on $C$. Let $N_{1}=\left\{x: x_{\alpha}=0\right.$ except for a countable set of $\left.\alpha\right\}$ and $N_{2}=\left\{x: x_{\alpha}=1\right.$ except for a countable set of $\left.\alpha\right\}$. It is easy to see that $N_{1}$ and $N_{2}$ bound $\mathfrak{A}$. Since $N_{1} \cap N_{2}$ is void, it follows that $\mathfrak{A}$ does not have a minimal boundary.

For an example of a function algebra whose minimal boundary is distinct from its Silov boundary, let $C$ be the subset $\{z:|z|=1\}$ of the complex plane and let $\mathfrak{A}$ consist of all continuous functions $f$ on $C$ which have the property that there exists a continuous function $\hat{f}$ on $\{z:|z| \leqq 1\}$ such that $\hat{f}(z)=f(z)$ for $z$ in $C$, such that $\hat{f}$ is analytic on $\{z:|z|<1\}$, and such that $\hat{f}(1)=\hat{f}(0)$. It is easy to see that $\mathfrak{A}$ is a separating Banach algebra of continuous functions on $C$. It is also not difficult to show that the Šilov boundary of $\mathfrak{A}$ is $C$, whereas the minimal boundary 
of $\mathfrak{A}$ is the set $\{z:|z|=1, z \neq 1\}=C-\{1\}$.

THeOREm 2. Let $\mathfrak{i}$ be a separating Banach algebra of continuous functions on a compact metrizable Hausdorff space C. For each positive integer $n$, let $U_{n}$ consist of all points $x$ in $C$ such that there exists $f$ in $\mathfrak{A}$ with $\|f\| \leqq 1,|f(x)|>3 / 4$, and $|f(y)|<1 / 4$ for all $y$ in $D_{n}(x)$, where $D_{n}(x)=\left\{y: \rho(x, y) \geqq n^{-1}\right\}$ and $\rho$ is a metric on $C$. Then $U_{n}$ is open and $\cap U_{n}=M$, where $M$ is the minimal boundary of $\mathfrak{A}$.

Proof. If $f$ is any function in $\mathfrak{A}$, it is clear that the set $\sigma_{n}(f)=$ $\left\{x: x \in C,|f(x)|>3 / 4,|f(y)|<1 / 4\right.$ whenever $\left.y \in D_{n}(x)\right\}$ is open for each $n$. Since $U_{n}$ is the union of the sets belonging to the class

$$
\left\{\sigma_{n}(f): f \in \mathfrak{A},\|f\| \leqq 1\right\},
$$

it follows that $U_{n}$ is open.

If $x \in M$, by Theorem 1 there exists $f$ in $\mathfrak{A}$ with $S(f)=\{x\}$. It is clearly no restriction to assume that $\|f\|=1$. Hence $|f(x)|=1$. Since $|f(y)|<1$ when $y$ is in the compact set $D_{n}(x)$, it follows that there exists a positive integer $p_{n}$ such that $|f(y)|^{p_{n}}<1 / 4$ when $y \in D_{n}(x)$. Thus $x \in \sigma_{n}\left(f^{p_{n}}\right)$. Therefore $x \in U_{n}$. Since this is true for each $n$, it follows that $x \in \bigcap U_{n}$. Therefore $M \subset \bigcap U_{n}$.

Now consider a fixed $x$ in $\bigcap U_{n}$. We must prove that $x \in M$. To this end, we construct by induction a sequence $\left\{g_{n}\right\}$ of functions in $\mathfrak{A}$ having the following properties:

(i) $\left\|g_{n+1}-g_{n}\right\| \leqq 2^{-n+1}$

(ii) $\left\|g_{n}\right\| \leqq 3\left(1-2^{-n-1}\right)$

(iii) $g_{n}(x)=3\left(1-2^{-n}\right)$

(iv) $\left|g_{n+1}(y)-g_{n}(y)\right|<2^{-n-1}$ if $y \in D_{n}(x)$.

We first construct $g_{1}$. Since $x \in U_{1}$, there exists a function $f$ in $\mathfrak{A}$ such that $\|f\| \leqq 1$ and $x \in \sigma_{1}(f)$. Let

$$
g_{1}=\frac{3}{2}[f(x)]^{-1} f .
$$

Since $|f(x)|>3 / 4$, we have $\left\|g_{1}\right\| \leqq 3 / 2 \cdot 4 / 3=2<3\left(1-2^{-2}\right)$, so that $g_{1}$ satisfies (ii). Clearly $g_{1}(x)=3\left(1-2^{-1}\right)$, so that $g_{1}$ satisfies (iii). Hence $g_{1}$ satisfies all of the relevant conditions. Assume now that $g_{1}, \cdots, g_{k}$ have been chosen to satisfy all of the relevant conditions. Since $g_{k}(x)=$ $3\left(1-2^{-k}\right)$, there exists an integer $j>k$ such that $\left|g_{k}(y)\right|<3\left(1-2^{-k}\right)+2^{-k-2}$ for $\rho(x, y)<j^{-1}$, i.e., for $y$ in $C-D_{j}(x)$. Since $x \in U_{j}$, there exists a function $f$ in $\mathfrak{A}$ such that $\|f\| \leqq 1$ and $x \in \sigma_{j}(f)$. Define $h=$ $3 \cdot 2^{-k-1}[f(x)]^{-1} f$. Thus $h(x)=3 \cdot 2^{-k-1}$. Since $\|f\| \leqq 1$ and $|f(x)|>3 / 4$, we see that $\|h\| \leqq 2^{-k+1}$. Since also $|f(y)|<1 / 4$ for $y$ in $D_{j}(x)$, we see 
that $|h(y)|<2^{-k-1}$ for $y$ in $D_{j}(x)$. Let $g_{k+1}=g_{k}+h$. It follows immediately that

$$
\text { (i) }\left\|g_{k+1}-g_{k}\right\| \leqq 2^{-k+1} \text {, }
$$

that

$$
\text { (iv) }\left|g_{k+1}(y)-g_{k}(y)\right|<2^{-k-1} \text { if } y \in D_{k}(x) \text {, }
$$

and that

$$
\text { (iii) } \begin{aligned}
g_{k+1}(x) & =g_{k}(x)+h(x)=3\left(1-2^{-k}\right)+3 \cdot 2^{-k-1} \\
& =3\left(1-2^{-k-1}\right) .
\end{aligned}
$$

If $y \in D_{j}(x)$, then

$$
\begin{aligned}
\left|g_{k+1}(y)\right| & \leqq\left|g_{k}(y)\right|+|h(y)| \\
& <|| g_{k} \|+2^{-k-1} \leqq 3\left(1-2^{-k-1}\right)+2^{-k-1} \\
& =3-2^{-k}<3\left(1-2^{-k-2}\right) .
\end{aligned}
$$

If $y \in C-D_{j}(x)$, then

$$
\begin{aligned}
\left|g_{k+1}(y)\right| & \leqq\left|g_{k}(y)\right|+|h(y)| \leqq 3\left(1-2^{-k}\right)+2^{-k-2}+\|h\| \\
& \leqq 3\left(1-2^{-k}\right)+2^{-k-2}+2^{-k+1}=3\left(1-2^{-k-2}\right) .
\end{aligned}
$$

It follows that

$$
\text { (ii) }\left\|g_{k+1}\right\| \leqq 3\left(1-2^{-k-2}\right) \text {. }
$$

Thus $g_{k+1}$ has the relevant properties. We have thus constructed the sequence $\left\{g_{n}\right\}$. By condition (i), the sequence $\left\{g_{n}\right\}$ converges uniformly on $C$ to a function $g$ in $\mathfrak{A}$. By (ii), $\|g\| \leqq 3$. By (iii), $g(x)=3$. If $y \in D_{n}(x)$, then

$$
|g(y)| \leqq\left\|g_{n}\right\|+\sum_{k=n}^{\infty}\left|g_{k+1}(y)-g_{k}(y)\right|<3\left(1-2^{-n-1}\right)+\sum_{k=n}^{\infty} 2^{-k-1}<3 .
$$

Thus $S(g)=\{x\}$. Therefore $x \in M$, as was to be proved.

COROLlaRY. If $\mathfrak{A}$ is a separating Banach algebra of continuous functions on a compact metrizable Hausdorff space $C$, then the minimal boundary $M$ of $\mathfrak{A}$ is a countable intersection of open sets.

\section{Representation by measures.}

We now prove the fundamental result of this paper.

THEOREM 3. Let $\mathfrak{A}$ be a separating Banach algebra of continuous functions on a compact metrizable Hausdorff space $C$. Let $\mathfrak{A}$ contain the function 1 , Let $x$ be a point of $C-M$, where $M$ is the minimal 
boundary of $\mathfrak{H}$. Then there exists a non-negative Borel measure $\gamma$ of norm 1 on $C-\{x\}$ such that $f(x)=\int f d \gamma$ for all $f$ in $\mathfrak{N}$.

Proof. We assume that a metric $\rho$ on $C$ is given. For each positive integer $n$, let

$$
D_{n}=\left\{y: y \in C, \rho(x, y) \geqq n^{-1}\right\} .
$$

Let $b$ and $c$ be real numbers such that $0<b<1 / 4<3 / 4<c<1$. For each positive integer $n$ and each positive integer $m$, let $h_{n m}$ be a continuous function on $C$ such that $h_{n m}(y)=b^{1 / m}$ for $y \in D_{n}, h_{n m}(y)=c^{1 / m}$ for $y \in C-D_{2 n}$, and $b^{1 / m} \leqq h_{n m}(y) \leqq c^{1 / m}$ for all $y$. Such a function exists because the closures of the sets $D_{n}$ and $C-D_{2 n}$ are disjoint. There are two cases to consider. Either there exists (Case 1) for each positive integer $n$ a positive integer $m$ and a function $f$ in $\mathscr{A}$ such that $|f(x)|>(3 / 4)^{1 / m}$ and $|f(y)| \leqq h_{n m}(y)$ for all $y$ in $C$, or (Case 2) there exists a positive integer $n$ such that for all positive integers $m$ and for all $f$ in $\mathfrak{N}$ either $|f(x)| \leqq(3 / 4)^{1 / m}$ or $|f(y)|>h_{n m}(y)$ for some $y$ in $C$. We shall show that Case 1 is impossible and that Case 2 implies the theorem to be proved.

Assume now that Case 1 obtains. Let the positive integer $n$ be given, and choose $f$ in $\mathfrak{A}$ and a positive integer $m$ such that $|f(x)|>(3 / 4)^{1 / m}$ and $|f(y)| \leqq h_{n m}(y)$ for all $y$.

Write $g=f^{m}$. Since $|f(y)| \leqq h_{n m}(y) \leqq c^{1 / m}$ for all $y$, we have $|g(y)| \leqq c$ for all $y$. Thus $\|g\| \leqq c<1$. Since $|f(x)|>(3 / 4)^{1 / m}$ we have $|g(x)|>3 / 4$. Since $|f(y)| \leqq h_{n m}(y)=b^{1 / m}$ for $y$ in $D_{n}$, we have $|g(y)| \leqq$ $b<1 / 4$ for $y$ in $D_{n}$. It follows that $x \in U_{n}$, where $U_{n}$ is the set defined in Theorem 2. Since this is true for each $n$, we have $x \in \cap U_{n}=M$, by Theorem 2. This contradicts the hypothesis of Theorem 3 . Therefore Case 1 is impossible.

We are therefore justified in assuming that Case 2 obtains. Thus there exists a positive integer $n$, henceforth fixed, such that for all positive integers $m$ and all $f$ in $\mathfrak{A}$ either $|f(x)| \leqq(3 / 4)^{1 / m}$ or $|f(y)|>h_{n m}(y)$ for some $y$ in $C$. Consider now a positive integer $m$. For each $f$ in $\mathfrak{A}$ either $|f(x)| \leqq(3 / 4)^{1 / m}$ or $\left\|f h^{-1}\right\|>1$, where $h=h_{n m}$. Thus $|f(x)| \leqq$ $(3 / 4)^{1 / m}$ whenever $f \in \mathfrak{A}$ and $\left\|f h^{-1}\right\| \leqq 1$. Let $\mathfrak{B}$ be the Banach space of all continuous functions on $C$, under the uniform norm, and let $\mathfrak{B}_{0}$ be the subspace $\left\{f h^{-1}: f \in \mathfrak{A}\right\}$ of $\mathfrak{B}$. Define the linear functional $\varphi$ on $\mathfrak{B}_{0}$ by defining $\varphi\left(f h^{-1}\right)=f(x)$ for each $f$ in $\mathfrak{A}$. Since $|f(x)| \leqq(3 / 4)^{1 / m}$ if $f \in \mathfrak{A}$ and $\left\|f h^{-1}\right\| \leqq 1$, it follows that $\|\mathcal{\|}\|(3 / 4)^{1 / m}$. By the HahnBanach theorem, there exists an extension $\rho_{0}$ of $\rho$ which is a linear functional on $\mathfrak{B}$ with $\left\|\varphi_{0}\right\| \leqq(3 / 4)^{1 / m}$. By the Riesz representation theorem, there exists a measure $\nu_{m}$ on $C$ such that $\left\|\nu_{m}\right\| \leqq(3 / 4)^{1 / m}$ and $\mathcal{P}_{0}(f)=\int f d \nu_{m}$ for all continuous functions $f$ on $C$. Thus 


$$
f(x)=\varphi\left(f h^{-1}\right)=\varphi_{0}\left(f h^{-1}\right)=\int f h^{-1} d \nu_{m}
$$

for all $f$ in $\mathfrak{A}$. If we define the measure $\mu_{m}$ by

$$
\mu_{m}(S)=\int_{S} h^{-1} d \nu_{m}
$$

for all Borel subsets $S$ of $C$, it follows that $f(x)=\int f d \mu_{m}$ for all $f$ in গ. In particular, $1=\int d \mu_{m}$. Thus $\left\|\mu_{m}\right\| \geqq 1$. Let the measure $\nu_{m}^{0}$, the restriction of $\nu_{m}$ to the set $D_{2 n}$, be defined by $\nu_{m}^{0}(S)=\nu_{m}\left(S \cap D_{2 n}\right)$ for each Borel set $S$. Let $\nu_{m}^{1}$, the restriction of $\nu_{m}$ to $C-D_{2 n}$, be defined similarly. Thus

$$
\left\|\nu_{m}^{0}\right\|+\left\|\nu_{m}^{1}\right\|=\left\|\nu_{m}\right\| \leqq\left(\frac{3}{4}\right)^{1 / m}
$$

Similarly, let $\mu_{m}^{0}$ be the restriction of $\mu_{m}$ to $D_{2 n}$, and let $\mu_{m}^{1}$ be the restriction of $\mu_{m}$ to $C-D_{2 n}$. Thus

$$
\left\|\mu_{m}^{0}\right\|+\left\|\mu_{m}^{1}\right\|=\left\|\mu_{m}\right\| \geqq 1 .
$$

Since $[h(y)]^{-1}=c^{-1 / m}$ for all $y$ in $C-D_{2 n}$ and since $\mu_{m}^{1}(S)=\int_{S-D_{2 n}} h^{-1} d \nu_{m}$, for all Borel sets $S$, we see that $\mu_{m}^{1}=c^{-1 / m} \nu_{m}^{1}$, so that $c^{1 / m}\left\|\mu_{m}^{1}\right\|=\left\|\nu_{m}^{1}\right\|$. Since $|h(y)|^{-1} \leqq b^{-1 / m}$ for all $y$, and therefore for all $y$ in $D_{2 n}$, we see similarly that $b^{1 / m}\left\|\mu_{m}^{0}\right\| \leqq\left\|\nu_{m}^{0}\right\|$. Thus

$$
b^{1 / m}\left\|\mu_{m}^{0}\right\|+c^{1 / m}\left\|\mu_{m}^{1}\right\| \leqq\left\|\nu_{m}^{0}\right\|+\left\|\nu_{m}^{1}\right\| \leqq\left(\frac{3}{4}\right)^{1 / m} .
$$

Combined with the inequality

$$
b^{1 / m}\left\|\mu_{m}^{0}\right\|+b^{1 / m}\left\|\mu_{m}^{1}\right\| \geqq b^{1 / m},
$$

this gives

$$
\left[c^{1 / m}-b^{1 / m}\right]\left\|\mu_{m}^{1}\right\| \leqq\left(\frac{3}{4}\right)^{1 / m}-b^{1 / m}
$$

Thus

$$
\begin{gathered}
\left\|\mu_{m}^{1}\right\| \leqq\left[\left(\frac{3}{4}\right)^{1 / m}-b^{1 / m}\right]\left[c^{1 / m}-b^{1 / m}\right]^{-1} \\
\text { Since }\left\|\mu_{m}\right\|=\left\|\mu_{m}^{0}\right\|+\left\|\mu_{m}^{1}\right\| \leqq b^{-1 / m}\left\|\nu_{m}^{0}\right\|+b^{-1 / m}\left\|\nu_{m}^{1}\right\| \\
\\
\leqq b^{-1 / m}\left\|\nu_{m}\right\| \leqq b^{-1 / m},
\end{gathered}
$$

there exists a subsequence $\left\{\mu_{m_{i}}\right\}$ of $\left\{\mu_{m}\right\}$ which converges in the weak star topology for measures on $C$ to a measure $\mu$ on $C$ with $\|\mu\| \leqq 1$. Also, 


$$
\int f d \mu=\lim \int f d \mu_{m_{i}}=f(x)
$$

for each $f$ in $\mathfrak{A}$.

Since $C-D_{2 n}$ is open and since

$$
\left\|\mu_{m}^{1}\right\| \leqq\left[\left(\frac{3}{4}\right)^{1 / m}-b^{1 / m}\right]\left[c^{1 / m}-b^{1 / m}\right]^{-1}
$$

for each $m$, we have

$$
\left\|\mu^{1}\right\| \leqq \lim \left[\left(\frac{3}{4}\right)^{1 / m}-b^{1 / m}\right]\left[c^{1 / m}-b^{1 / m}\right]^{-1},
$$

where $\mu^{1}$ is the restriction of $\mu$ to $C-D_{2 n}$. Now

$$
\begin{aligned}
& \lim _{h \rightarrow 0}\left[\left(\frac{3}{4}\right)^{h}-b^{h}\right]\left[c^{h}-b^{h}\right]^{-1} \\
& \quad=\lim _{h \rightarrow 0}\left[\left(\ln \frac{3}{4}\right)\left(\frac{3}{4}\right)^{h}-(\ln b) b^{h}\right]\left[(\ln c) c^{h}-(\ln b) b^{h}\right]^{-1} \\
& \quad=\left[\ln \frac{3}{4}-\ln b\right][\ln c-\ln b]^{-1}=\ln \left(\frac{3}{4} b^{-1}\right)\left[\ln \left(c b^{-1}\right)\right]^{-1}<1 .
\end{aligned}
$$

Thus, if $\mu^{2}$ denotes the restriction of $\mu$ to the set $\{x\}$, we have $\left\|\mu^{2}\right\| \leqq$ $\left\|\mu^{1}\right\|<1$. Thus there exists a constant a with $|a|<1$ such that $\int f d \mu^{2}=a f(x)$ for all continuous functions $f$ on $C$. Let $\mu^{3}$ be the restriction of $\ell^{\prime}$ to $C-\{x\}$, so that $\mu^{2}+\mu^{3}=\mu$ and $\left\|\mu^{2}\right\|+\left\|\ell^{3}\right\|=\|\mu\| \leqq 1$. Thus

$$
\int f d \mu=\int f d \mu_{2}+\int f d \mu_{3}=a f(x)+\int f d \mu_{3}
$$

for all $f$ in $\mathfrak{Y}$. Therefore $(1-a) f(x)=\int f d \mu_{3}$ for all $f$ in $\mathscr{N}$. Since $\left\|\mu^{3}\right\| \leqq 1-\left\|\mu_{2}\right\|=1-|a|$, and since $1 \in \mathfrak{A}$, we have $1-|a| \leqq|1-a|=$ $\left|\int 1 d \mu_{3}\right| \leqq\left\|\mu_{3}\right\| \leqq 1-|a|$. Thus $a$ is positive. Define $\gamma=(1-a)^{-1} \mu_{3}$. Thus

$$
f(x)=(1-a)^{-1} \int f d \mu_{3}=\int f d \gamma
$$

for all $f$ in $\mathfrak{A}$. Also, $\|\gamma\| \leqq(1-a)^{-1}\left\|\ell_{3}\right\| \leqq 1$. Since $1 \in \mathfrak{U}$ we have $1=\int d \gamma$. Therefore $\gamma$ is a non-negative valued measure. This completes the proof of the theorem.

COROLlary. Let $\mathfrak{H}$ be a separating Banach algebra containing the 
unit function of continuous functions on the compact metrizable Hausdorff space $C$. Let $\Re_{0}$ consist of all functions on $C$ which are real parts of functions in $\mathfrak{U}$, and let $\mathfrak{R}$ be the uniform closure of $\Re_{0}$. Let $M_{0}$ consist of all points $x$ in $C$ such that there exists $f$ in $\mathfrak{R}$ with $|f(x)|>|f(y)|$ for all $y \neq x$ in $C$. Then $M_{0}$ equals the minimal boundary $M$ of $\mathfrak{A}$.

Proof. If $x \in M$, there exists $g$ in $C$ such that $|g(x)|>|g(y)|$ for all $y \neq x$ in $C$. It is no loss of generality to assume that $g(x)=1$. If we let $f$ be the real part of $g$, then $f \in \Re_{0} \subset \Re$ and $|f(x)|=|g(x)|>$ $|g(y)| \geqq|f(y)|$ for all $y \neq x$. Hence $x \in M_{0}$.

If $x$ is not in $M$, then there exists a real-valued measure $\gamma$ on $M-\{x\}$ of norm 1 such that $g(x)=\int g d \gamma$ for all $g$ in $\mathfrak{A}$, by Theorem 3. Since $\gamma$ is real-valued, it follows that $f(x)=\int f d \gamma$ for all $f$ in $\Re_{0}$. Thus $f(x)=\int f d \gamma$ for all $f$ in $\Re$. If $x$ where in $M_{0}$, there would exist $f$ in $\Re$ with $1=f(x)>|f(y)|$ for all $y \neq x$. Thus $1=f(1)=\int f d \gamma \leq$ $\left|\int f d \gamma\right|<1$, since $|f(y)|<1$ for $y \neq x$ and since $\|\gamma\|=1$. This contradiction shows that $x$ is not $M_{0}$. Hence $M=M_{0}$, as was to be proved.

DeLeeuw has found a proof of Theorem 3 which is somewhat simpler than the one given here.

4. Applications. We now apply the results of the previous sections to certain problems of approximation in one complex variable.

Definition 3. Let $C$ be a compact subset of the complex plane. Then $\Lambda_{0}(C)$ will consist of all continuous functions on $C$ which are analytic at interior points of $C$, and $\Lambda_{1}(C)$ will consist of all continuous functions on $C$ which can be uniformly approximated arbitrarily closely by rational functions whose poles lie in the complement of $C$.

It is clear that $\Lambda_{1}(C) \subset \Lambda_{0}(C)$, and that $\Lambda_{0}(C)$ and $\Lambda_{1}(C)$ are separating Banach algebras of continuous functions on $C$. Mergelyan [2] has shown that $\Lambda_{0}(C)=\Lambda_{1}(C)$ in case the complement of $C$ consists of only a finite number of components. No necessary and sufficient condition is known that $\Lambda_{1}(C)=\Lambda_{0}(C)$. In case $C$ has no interior, we shall obtain in Theorem 5 below a necessary and sufficient condition that every continuous function on $C$ be uniformly approximable by rational functions with poles in $-C$.

THEOREM 4. Let $C$ be a compact subset of the complex plane with no interior and let $M$ be the minimal boundary of $\Lambda_{1}(C)$. Then either 
$\Lambda_{1}(C)=\Lambda_{0}(C)$ or $C-M$ has positive 2-dimensional Lebesgue measure.

Proof. Assume that $\Lambda_{1}(C) \neq \Lambda_{0}(C)$. We must show that $C-M$ has positive 2-dimensional Lebesgue measure. Now $\Lambda_{0}(C)$ is the Banach space of all continuous complex-valued functions on $C$, and $\Lambda_{1}(C)$ is a proper subspace, since $\Lambda_{1}(C) \neq \Lambda_{0}(C)$. By the Hahn-Banach theorem, there exists a continuous linear functional $\varphi \neq 0$ on $\Lambda_{0}(C)$ which vanishes on $\Lambda_{1}(C)$. By the Riesz representation theorem, there exists a finite complex-valued Borel measure $\mu$ on $C$ which represents $\varphi$. Thus $\mu \neq 0$ and $\int f d \mu=0$ for all $f$ in $\Lambda_{1}(C)$. In particular, $\int(z-\zeta)^{-1} d \mu(\zeta)=0$ whenever $z$ is not in $C$, since the function $(z-\zeta)^{-1}$ is a rational function of $\zeta$ whose pole, $z$, is not in $C$. Since the function $z^{-1}$ of $z$ is integrable with respect to Lebesgue measure $d x d y$ over any finite region of the plane, and since $\mu$ is a finite measure on the compact set $C$, the integral

$$
h(z)=\int(z-\zeta)^{-1} d \mu(\zeta)
$$

will exist for almost all values of $z$, and the function $h(z)$ so defined, called the convolution of the measure $\mu$ and the function $z^{-1}$ and written $h=z^{-1} * \mu$, will be integrable with respect to Lebesgue measure over any finite region of the plane. Since we have seen above that $h(z)=0$ if $z$ is not in $C$, it follows that $h$ is integrable.

Assume that the integrable function $h$ vanishes almost everywhere, so that the integral $h(z)=\int(z-\zeta)^{-1} d \mu(\zeta)$ exists and vanishes for almost all $z$. To obtain a contradiction from this assumption, we use the equation $\frac{\partial}{\partial z^{*}} \frac{1}{z}=\pi \delta([7]$ p. 49) from the theory of distributions. This means that for any function $g$ on the complex plane which vanishes in a neighborhood of $\infty$ and which has continuous partial derivatives of all orders we have

$$
-\iint(z-\zeta)^{-1} \frac{1}{2}\left(\frac{\partial}{\partial x}+i \frac{\partial}{\partial y}\right) g(z) d x d y=\pi g(\zeta)
$$

for all values of $\zeta$. If we write $g_{1}(z)=\frac{1}{2}\left(\frac{\partial}{\partial x}+i \frac{\partial}{\partial y}\right) g(z)$ and integrate both sides of the above equation with respect to $\mu$ we obtain

$$
\begin{aligned}
\pi \int g(\zeta) d \mu(\zeta) & =-\int\left\{\iint(z-\zeta)^{-1} g_{1}(z) d x d y\right\} d \mu(\zeta) \\
& =-\iint g_{1}(z)\left\{\int(z-\zeta)^{-1} d \mu(\zeta)\right\} d x d y=0,
\end{aligned}
$$


since $h(z)$ vanishes almost everywhere. The use of Fubini's theorem is justified since $z-\zeta$, and therefore $(z-\zeta)^{-1}$, is measurable with respect to the product of the measures $g_{1}(z) d x d y$ and $d \mu(\zeta)$ and since

$$
\int\left\{\iint\left|g_{1}(z)\right||z-\zeta|^{-1} d x d y\right\}|d \mu(\zeta)|
$$

is finite. Now every continuous function $g_{0}$ on $C$ can be uniformly approximated by such functions $g$, so that $\int g_{0}(\zeta) d \mu(\zeta)=0$. By the uniqueness part of the Riesz representation theorem, it follows that $\mu=0$. This contradiction shows that there exists a set $\Gamma$ of $C$ of positive Lebesgue measure such that the integral $h(z)$ exists and does not vanish for all $z$ in $\Gamma$. We may clearly assume that at no point of $\Gamma$ does $\mu$ have point mass.

Let $z_{0}$ be any point in $\Gamma$, so that $h\left(z_{0}\right)=c$ exists and is not zero. Let $f$ be any function in $\Lambda_{1}(C)$ such that $f\left(z_{0}\right)=0$. Let $\left\{f_{n}\right\}$ be a sequence of rational functions with poles in the complement of $C$ converging uniformly to $f$ on $C$. Since $f_{n}\left(z_{0}\right) \rightarrow f\left(z_{0}\right)=0$ as $n \rightarrow \infty$, we see that $\left\{g_{n}\right\}$ converges uniformly to $f$ on $C$, where $g_{n}=f_{n}-f_{n}\left(z_{0}\right)$. Thus $g_{n}$ is a rational function with poles in $-C$ which vanishes at $z_{0}$, so that there exists a rational function $g_{n}^{\prime}$ with poles in $-C$ such that $g_{n}(z)=$ $g_{n}^{\prime}(z)\left(z-z_{0}\right)$ for all $z$. Hence

$$
\int g_{n}(z)\left(z-z_{0}\right)^{-1} d \mu(z)=\int g_{n}^{\prime}(z) d \mu(z)=0
$$

for each $n$, since $g_{n}^{\prime} \in \Lambda_{1}(C)$. Passing to the limit, we see that

$$
\int f(z)\left(z-z_{0}\right)^{-1} d \mu(z)=0 \text {. }
$$

Since this is true for all $f$ in $\Lambda_{1}(C)$ with $f\left(z_{0}\right)=0$, it follows that for an arbitrary $f$ in $\Lambda_{1}(C)$ we have

$$
\begin{aligned}
& \int f(z)\left(z-z_{0}\right)^{-1} d \mu(z)=\int\left[f(z)-f\left(z_{0}\right)\right]\left(z-z_{0}\right)^{-1} d \mu(z) \\
& \quad+\int f\left(z_{0}\right)\left(z-z_{0}\right)^{-1} d \mu(z)=0+f\left(z_{0}\right)\left(-h\left(z_{0}\right)\right)=-c f\left(z_{0}\right) .
\end{aligned}
$$

If we let $\delta_{0}$ denote the measure of mass +1 at the point $z_{0}$, it follows that

$$
\nu=\left(z-z_{0}\right)^{-1} \mu+c \delta_{0}
$$

is a measure on $C$ which annihilates $\Lambda_{1}(C)$. Now if $z_{0}$ were in $M$, there would exist $f$ in $\Lambda_{1}(C)$ with $f\left(z_{0}\right)=1$ and $|f(z)|<1$ for all $z \neq z_{0}$ in $C$. Since $\nu\left(\left\{z_{0}\right\}\right)=c \neq 0$, it is clear that $\int f^{n} d \nu \neq 0$ if $n$ is sufficiently large. 
This is a contradiction, since $f^{n} \in \Lambda_{1}(C)$. This shows that $z_{0}$ is not in $M$. Since $z_{0}$ was any point in $I^{\prime}$ we have $I^{\prime} \subset C-M$. Since $I^{\prime}$ has positive measure, $C-M$ has positive measure, as was to be proved.

To restate the theorem, if for every point $z$ on $C$, with the possible exception of a set of measure 0 , there exists a continuous function $f$ on $C$ with $|f(z)|>|f(\zeta)|$ for all $\zeta \neq z$ in $C$ which can be uniformly approximated by rational functions with poles in $-C$, then every continuous function on $C$ can be uniformly approximated by rational functions whose poles lie in $-C$.

THeOREm 5. Let $C$ be a compact subset of the complex plane without interior. Let $\Lambda_{0}(C)$ be the algebra of all continuous complex-valued functions on $C$ and let $\Lambda_{1}(C)$ be those functions in $\Lambda_{v}(C)$ which can be uniformly approximated by rational functions with poles in $-C$, and let $M$ be the minimal boundary of $\Lambda_{1}(C)$. Let $\Delta_{0}(C)$ consist of all continuous real-valued functions on $C$, and let $\Delta_{1}(C)$ consist of all continuous real-valued functions on $C$ which are uniformly approximable by real parts of functions in $\Lambda_{1}(C)$. Let $M_{0}$ consist of all points $z$ in $C$ such that there exists $f$ in $\Delta_{1}(C)$ with $|f(z)|>|f(\zeta)|$ for all $\zeta \neq z$ in $C$. Then $M=M_{0}$ and the following statements are equivalent:

(i) $\Lambda_{1}(C)=\Lambda_{0}(C)$

(ii) $C-M$ has measure 0

(iii) $M=C$

(iv) $\Delta_{1}(C)=\Delta_{0}(C)$.

Proof. The fact that $M=M_{0}$ is a special casc of the corollary to Theorem 3. It is clear that (i) $\Rightarrow$ (iii) $\Rightarrow$ (ii). But (ii) $\Rightarrow$ (i) by Theorem 4. Thus (i), (ii), and (iii) are equivalent. It is also clear that (i) $\Rightarrow$ (iv). But (iv) implies that $M_{0}=C$. Thus (iv) $\Rightarrow$ (iii). This proves Theorem 5 .

Theorem 5 thus gives results concerning approximation on a nowhere dense subset of the complex plane by rational functions or by real parts of rational functions, and shows that the two problems are related. The results for approximation by the real parts of rational functions are similar in outward appearance to results of Brelot [2] and Deny [4], who consider approximation by functions harmonic in a neighborhood of $C$, but there does not seem to be an essential connection, due to the fact that a function harmonic in the neighborhood of $C$ need not be the real part of an analytic function, since the conjugate harmonic function might be multiple-valued.

\section{REFERENCES}

1. E. Bishop, Some theorems concerning function algebras, Bull. Amer. Math. Soc. 65 (1959), 77-78. 
2. M. Brelot, Critères de régularite et de stabilité, Acad. Roy. Belgique Bull. Cl. Sci. 25 (1939), 125-137.

3. G. Choquet, Existence des représentations intégrales au moyen des points extrémaux dans les cônes convexes, C. R. Acad. Sci. Paris 243 (1956), 699-702.

4. J. Deny, Sur l'approximation des fonctions harmoniques, Bull. Soc. Math. France 73 (1945), 71-73.

5. L. H. Loomis, Abstract harmonic analysis, New York, 1953.

6. S. N. Mergelyan, On the representation of functions by series of polynomials on closed sets, Amer. Math. Soc. Translation no. 85, 1953.

7. L. Schwartz, Théorie des distributions vol. 1, Actualities Scient. et Indust., Paris, 1951. UNIVERSITY OF CALIFORNIA

Berkeley, California 


\section{PACIFIC JOURNAL OF MATHEMATICS}

\section{EDITORS}

\section{David Gilbarg}

Stanford University Stanford, California

\section{R. A. Beaumont}

University of Washington

Seattle 5, Washington

\section{A. L. Whiteman}

University of Southern California Los Angeles 7, California

L. J. Paige

University of California

Los Angeles 24, California

\section{ASSOCIATE EDITORS}

E. F. BECKENBACH
C. E. BURGESS
E. HEWITT
A. HORN

A. HORN

\author{
V. GANAPATHY IYER \\ R. D. JAMES \\ M. S. KNEBELMAN \\ L. NACHBIN
}

I. NIVEN

T. G. OSTROM

H. L. ROYDEN

M. M. SCHIFFER
E. G. STRAUS

G. SZEKERES

F. WOLF

K. YOSIDA

\section{SUPPORTING INSTITUTIONS}

\author{
UNIVERSITY OF BRITISH COLUMBIA \\ CALIFORNIA INSTITUTE OF TECHNOLOGY \\ UNIVERSITY OF CALIFORNIA \\ MONTANA STATE UNIVERSITY \\ UNIVERSITY OF NEVADA \\ OREGON STATE COLLEGE \\ UNIVERSITY OF OREGON \\ OSAKA UNIVERSITY \\ UNIVERSITY OF SOUTHERN CALIFORNIA
}

\author{
STANFORD UNIVERSITY \\ UNIVERSITY OF TOKYO \\ UNIVERSITY OF UTAH \\ WASHINGTON STATE COLLEGE \\ UNIVERSITY OF WASHINGTON \\ * * * * \\ AMERICAN MATHEMATICAL SOCIETY \\ CALIFORNIA RESEARCH CORPORATION \\ HUGHES AIRCRAFT COMPANY \\ SPACE TECHNOLOGY LABORATORIES
}

Mathematical papers intended for publication in the Pacific Journal of Mathematics should be typewritten (double spaced), and the author should keep a complete copy. Manuscripts may be sent to any one of the four editors. All other communications to the editors should be addressed to the managing editor, L. J. Paige at the University of California, Los Angeles 24, California.

50 reprints per author of each article are furnished free of charge; additional copies may be obtained at cost in multiples of 50 .

The Pacific Journal of Mathematics is published quarterly, in March, June, September, and December. The price per volume (4 numbers) is $\$ 12.00$; single issues, $\$ 3.50$. Back numbers are available. Special price to individual faculty members of supporting institutions and to individual members of the American Mathematical Society: $\$ 4.00$ per volume; single issues, $\$ 1.25$.

Subscriptions, orders for back numbers, and changes of address should be sent to Pacific Journal of Mathematics, 2120 Oxford Street, Berkeley 4, California.

Printed at Kokusai Bunken Insatsusha (International Academic Printing Co., Ltd.), No. 6, 2-chome, Fujimi-cho, Chiyoda-ku, Tokyo, Japan.

PUBLISHED BY PACIFIC IOURNAL OF MATHEMATICS, A NON-PROFIT CORPORATION

The Supporting Institutions listed above contribute to the cost of publication of this Journal, but they are not owners or publishers and have no responsibility for its content or policies. 


\section{Pacific Journal of Mathematics}

\section{Vol. 9, No. $3 \quad$ July, 1959}

Errett Albert Bishop, A minimal boundary for function algebras . . . . . . . . . . . . 629

John W. Brace, The topology of almost uniform convergence . . . . . . . . . . . . 643

Cecil Edmund Burgess, Chainable continua and indecomposability .......... 653

L. Carlitz, Multiplication formulas for products of Bernoulli and Euler

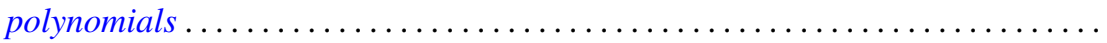

Eckford Cohen, A class of residue systems (mod $r$ ) and related arithmetical

functions. II. Higher dimensional analogues ....................

Shaul Foguel, Boolean algebras of projections of finite multiplicity . . . . . . . . . .

Richard Robinson Goldberg, Averages of Fourier coefficients .................

Seymour Goldberg, Ranges and inverses of perturbed linear operators .

Philip Hartman, On functions representable as a difference of convex functions ....

Milton Vernon Johns, Jr. and Ronald Pyke, On conditional expectation and

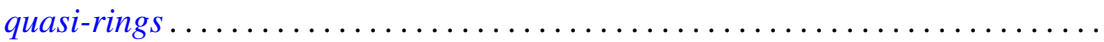

Robert Jacob Koch, Arcs in partially ordered spaces ....................

Gregers Louis Krabbe, A space of multipliers of type $L^{p}(-\infty, \infty) \ldots \ldots \ldots \ldots$

John W. Lamperti and Patrick Colonel Suppes, Chains of infinite order and their

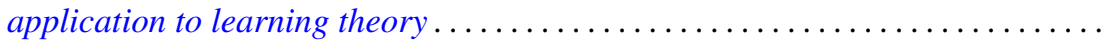

Edith Hirsch Luchins, On radicals and continuity of homomorphisms into Banach

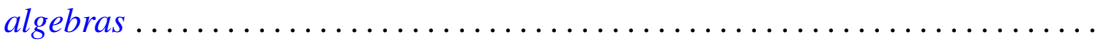

T. M. MacRobert, Multiplication formulae for the E-functions functions of their parameters.

Michael Bahir Maschler, Classes of minimal and representative domains and their kernel functions.

William Schumacher Massey, On the imbeddability of the real projective spaces in Euclidean space.

Thomas Wilson Mullikin, Semi-groups of class $\left(C_{0}\right)$ in $L_{p}$ determined by parabolic differential equations

Steven Orey, Recurrent Markov chains

Ernest Tilden Parker, On quadruply transitive groups ........ . .

Calvin R. Putnam, On Toeplitz matrices, absolute continuity, and unitary

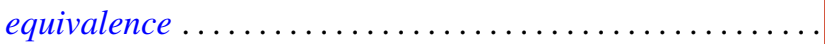

Helmut Heinrich Schaefer, On nonlinear positive operators.

Robert Seall and Marion Wetzel, Some connections between continued fractions and convex sets

Robert Steinberg, Variations on a theme of Chevalley

Olga Taussky and Hans Zassenhaus, On the similarity transformation between a

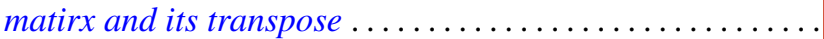

Emery Thomas, The suspension of the generalized Pontrjagin cohomology

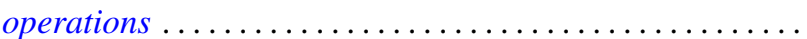

Joseph L. Ullman, On Tchebycheff polynomials ..................... 913

Richard Steven Varga, Orderings of the successive overrelaxation scheme ........ 925

Orlando Eugenio Villamayor, Sr., On weak dimension of algebras . 